\title{
Modularity of the rodent mandible: Integrating bones, muscles, and teeth
}

\author{
Miriam Leah Zelditch,a,* Aaron R. Wood, ${ }^{a}$ Ronald M. Bonett, ${ }^{b, c}$ and Donald L. Swiderskid,e \\ ${ }^{a}$ Museum of Paleontology, University of Michigan, Ann Arbor, MI 48109, USA \\ bepartment of Molecular, Cellular and Developmental Biology, University of Michigan, Ann Arbor, MI 48109, USA \\ 'Department of Biological Sciences, University of Tulsa, Tulsa, OK 74104, USA \\ ${ }^{d}$ Kresge Hearing Research Institute, University of Michigan, Ann Arbor, MI 48109, USA \\ ${ }^{\mathrm{e}}$ Museum of Zoology, University of Michigan, Ann Arbor, MI 48109, USA \\ *Author for correspondence (email: zelditch@umich.edu)
}

\begin{abstract}
SUMMARY Several models explain how a complex integrated system like the rodent mandible can arise from multiple developmental modules. The models propose various integrating mechanisms, including epigenetic effects of muscles on bones. We test five for their ability to predict correlations found in the individual (symmetric) and fluctuating asymmetric (FA) components of shape variation. We also use exploratory methods to discern patterns unanticipated by any model. Two models fit observed correlation matrices from both components: (1) parts originating in same mesenchymal condensation are integrated, (2) parts developmentally dependent on the same muscle form an integrated complex as do those dependent on teeth. Another fits the correlations observed in FA: each muscle insertion site is
\end{abstract}

an integrated unit. However, no model fits well, and none predicts the complex structure found in the exploratory analyses, best described as a reticulated network. Furthermore, no model predicts the correlation between proximal parts of the condyloid and coronoid, which can exceed the correlations between proximal and distal parts of the same process. Additionally, no model predicts the correlation between molar alveolus and ramus and/ or angular process, one of the highest correlations found in the FA component. That correlation contradicts the basic premise of all five developmental models, yet it should be anticipated from the epigenetic effects of mastication, possibly the primary morphogenetic process integrating the jaw coupling forces generated by muscle contraction with those experienced at teeth.

\section{INTRODUCTION}

Developmental modules are spatially delimited cell populations, internally coordinated in response to epigenetic interactions and quasi-autonomous with respect to pattern formation and differentiation (e.g., Raff 1996; Wolf et al. 2001). Modularity is widely viewed as fundamental to the evolution of complex phenotypes because it allows individual parts to evolve without inferring with others (Bolker 2000; Gerhart and Kirschner 2007; e.g., Needham 1933; Raff 1996; Wagner et al. 2007). Yet, even though autonomy is the defining criterion for modules, structures derived from different modules are often highly correlated, such as fore and hindlimbs (Hallgrímsson et al. 2002; Young and Hallgrimsson 2005), brain and skull (Richtsmeier et al. 2006) and parts of the mandible arising from different mesenchymal condensations (e.g., Atchley 1983; Atchley et al. 1985; Cheverud et al. 1991). Correlations among developmental modules are expected to arise when several modules combine to form a functionally integrated complex because functionally coupled traits are expected to become genetically integrated and thus develop and evolve in association with each other, independent of functionally independent traits (e.g., Lande 1980;
Cheverud 1982, 1984; Wagner 1988, 1996; Wagner et al. 2005). How modules are combined or even repartitioned during development remains largely mysterious even though those mechanisms may determine how readily patterns of integration evolve to match the structure of functional coupling (Klingenberg 2004, 2005), as well as whether developmentally canalized structures are weakly or highly integrated (Hallgrímsson et al. 2007a, b) or even whether the integration of functionally coupled structures is built into a developmental system.

The present study focuses on the mammalian mandible, a favored model system for studies of modularity and integration (e.g., Atchley and Hall 1991; Cheverud et al. 1991; Hall 2003; Klingenberg et al. 2003; Badyaev et al. 2005; Monteiro et al. 2005; Young and Badyaev 2006; Pavlicev et al. 2008). Various mechanisms have been hypothesized to explain mandibular integration, including variation in genes expressed in two or more mesenchymal condensations, such as those expressed by secondary chondroblasts, which could correlate coronoid, condyloid, and angular traits (Atchley and Hall 1991; Hall and Miyake 2000). Correlations such as these, which arise from common responses to a factor acting along parallel lines within different modules, exemplify parallel vari- 
ation (Klingenberg and Zaklan 2000; Klingenberg et al. 2001, 2004, 2003). A fundamentally different cause of integration depends not on the source of variation but rather on the route by which variation is transmitted along or between developmental pathways; these correlations require direct interactions, such as partitioning of a precursor or inductive signaling interactions (Klingenberg and Zaklan 2000; Klingenberg et al. 2001, 2004, 2003). Integration due to direct interactions may be more difficult to modify by natural selection because doing so might require restructuring developmental pathways (Klingenberg 2004, 2005). But direct interactions between functionally coupled parts could also ensure that their integration is built into a developmental system.

Muscle-bone interactions exemplify a case of direct interactions between functionally coupled traits that may build their integration into the developmental system. From a functional perspective, muscles and bones are parts of the same functional system in that bones provide skeletal struts and levers moved by forces supplied by muscles (Herring 1994). From a developmental perspective, muscles and bones are also tightly integrated, as evident from the skeletal anomalies produced in the absence of embryonic muscle contractions: bones and cartilages either fail to form at all or are greatly reduced and misshapen (e.g., Herring and Lakars 1981; Hall and Herring 1990; Rot-Nikcevic et al. 2006). Not surprisingly, these interactions figure prominently in models for mandibular integration (e.g., Atchley and Hall 1991; Cheverud et al. 1991; Klingenberg et al. 2003; Badyaev and Foresman 2004; Monteiro et al. 2005; Young and Badyaev 2006). These interactions can induce integration by both parallel variation and direct interactions according to whether the correlations are caused by variation in either muscle or bone genotype, or from signaling interactions between the two tissues due to strains resulting from muscle function.

To distinguish integration due to parallel variation from integration due to direct interactions, we use the ingenious approach devised by Klingenberg (Klingenberg and Zaklan 2000; Klingenberg et al. 2001, 2003). This approach is based on the rationale that the two sides of a bilaterally symmetric structure have the same genotype and develop within the same environment. Consequently, correlations between random deviations from bilateral symmetry, i.e., correlated fluctuating asymmetries (FA), cannot result from parallel variation but must come from direct interactions. To test hypotheses about the causes of these correlations, as well as to explore their structure for unanticipated correlations, we use the method pioneered by Monteiro et al. (2005) which makes it possible to examine correlations among complex, multidimensional traits. We first test five a priori hypotheses, three of which concern epigenetic interactions between bone and muscle. The three hypotheses that incorporate muscle-bone interactions differ regarding their predictions for the spatial extent of integration caused by these interactions. Unfortu- nately, none is based on mechanics of mastication and biting and no realistic hypothesis derived from those mechanics can be derived at present because we have no realistic models for the spatial distribution of masticatory strains across the rodent mandible. We, therefore, also use exploratory methods to examine the correlation structure of symmetric and FA components of mandibular shape variation.

\section{MATERIALS AND METHODS}

\section{Sample}

The 91 deer mice (Peromyscus maniculatus bairdii) analyzed in this study come from a sample previously used to examine the impact of dietary consistency on skull shape (Myers et al. 1996). All 91 come from the control group, which was fed standard pellets of Purina mouse chow and water, ad libitum. The mice comprise the second-generation born in the laboratory, bred from offspring of pregnant females captured near Ann Arbor (southeast Michigan). Mice were sacrificed at 50 days of age, by which point they had reached adult size and were capable of reproduction.

Mandibles were cleaned and separated into right and left hemimandibles, and each hemimandible was photographed twice in lateral view. Specimens were repositioned between photographs to assess measurement error due to both positioning and digitizing. Thirteen landmarks were digitized on each jaw (Fig. 1). Except for the landmark at the mental foramen (\#3) and the anterior of the masseteric ridge (\#4) all the landmarks are along the outline of the jaw. Because the 13 landmarks provide little information about jaw curvature, additional points were sampled along curves of the incisor alveolus, ventral ramus, and mandibular processes. These additional points (semi-landmarks) are not discrete anatomical loci and contain less information than landmarks because their spacing along the curve is arbitrary. However, semi-landmarks make it possible to study complex curving morphologies where landmarks are sparse. Thirty evenly spaced points were digitized along each curve using the "resample" function of the curve tracing tool in tpsDig2.1 (Rohlf 2006). Such a high density of points is needed to model the shape of the curve, which is part of the process of superimposing semi-landmarks; approximately half of the semilandmarks were removed from the analysis following superimposition, leaving a total of 98 points (Fig. 2).

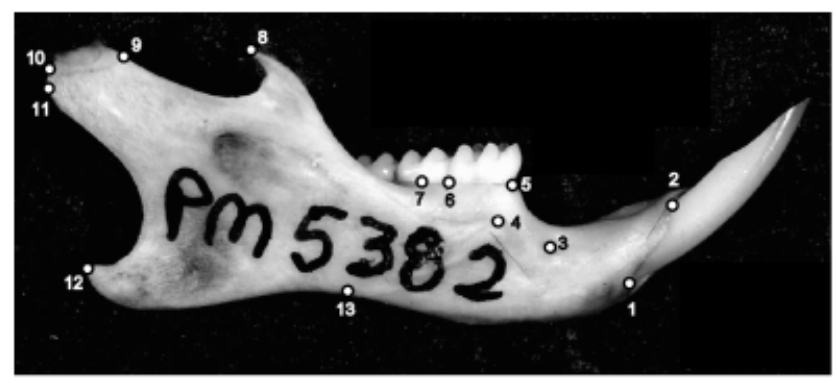

Fig. 1. Landmarks shown on a photograph of the mandible of the deer mouse, Peromyscus maniculatus bairdii. 


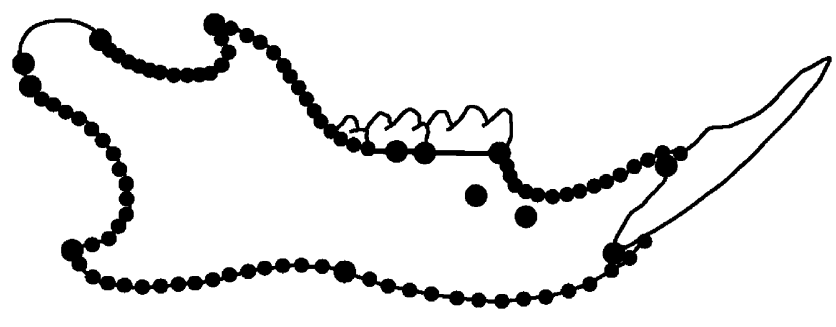

Fig. 2. Schematic of the deer mouse mandible showing landmarks (large points) and semi-landmarks (small points).

Three curves sampled by semi-landmarks are anchored by only one landmark. Of these, two capture the form of the incisor alveolus (dorsally and ventrally). Rather than beginning these curves at landmarks 1 and 2, where the incisors erupt from the alveolus laterally, the initial points are taken more medially because the curve being traced is medial to the opening of the alveoli. The other curve anchored by only one landmark measures the anterior coronoid, from the point at which the base can be distinguished from the molar alveolus, extending to the tip (\# 8). Remaining curves are anchored by landmarks at both ends.

\section{Superimposition and analysis of FA}

After reflecting coordinates for the left side, the two sides were combined and superimposed to remove variation in position, orientation, and scale. The superimposition of semi-landmarks requires specialized methods because they contain an aspect of variation unrelated to shape: their spacing along the curve. This variation is eliminated by sliding the semi-landmarks along their respective curves to match the positions of the corresponding points in the reference configuration, typically the mean shape (Adams et al. 2004; Sheets et al. 2004). Currently, there are two main methods for sliding semi-landmarks: the first minimizing the bending-energy of the thin-plate spline (Green 1996; Bookstein 1997; Gunz et al. 2005), the second minimizing the Procrustes distance from the mean shape (Sampson et al. 1996; Andresen et al. 2000; Bookstein et al. 2002). We use the second method because the Procrustes distance is the metric underlying the general theory of shape. According to this method, the tangent to the curve at each semi-landmark is estimated and then each semi-landmark is slid toward the normal of its respective tangent, minimizing the overall difference from the reference. Superimposition of semi-landmarks was done in Semiland (Sheets 2003), which also does a conventional least squares superimposition of the landmarks. Following superimposition, the coordinates of semi-landmarks can be used in any conventional shape analysis, provided that statistical tests take into account that they have only one degree of freedom. The overall dimensionality of the data containing a mix of landmarks and semi-landmarks is $2 K+L-4$, where $K$ is the number of landmarks and $L$ is the number of semi-landmarks.

The analysis of FA for geometric shape uses the standard twofactor mixed-model analysis of variance, with "individual" and "sides" as the two main factors (Leamy 1984; Palmer and Strobeck 1986). Symmetric variation among individuals is quantified by the main effect of "individuals" after correction for asymmetry; the consistent difference between sides (directional asymmetry) is quantified by the main effect of "sides." The interaction between the two main factors estimates the random deviations from bilateral symmetry, that is, FA. The statistical significance of FA is assessed by the F-ratio between the interaction and measurement error mean squares (Leamy, 1984; Palmer and Strobeck, 1986). This statistical approach has been adapted to the analysis of shape using Procrustes-based methods (Auffray et al. 1996; Klingenberg and McIntyre 1998; Klingenberg et al. 2002). For Procrustes shape data, the sum of squares for each effect is obtained by summing the sums of squares for each coordinate over all landmarks and semilandmarks, and the mean square is obtained by dividing that sum of squares by the degrees of freedom, which is equal to the univariate degrees of freedom for that effect multiplied by $2 K+L-4$. To test the statistical significance of FA, we used a permutation test. Analysis of FA was conducted in Sage (Marquez 2007b), which also calculates the shape coordinates for the symmetric and FA components of shape used in subsequent analyses. Preliminary analysis found a statistically significant among-family component of the variation in FA $(P<0.001)$, which was removed from the data by adding each individual's residuals from the mean for their family to the mean of the sample to ensure that we have controlled for parallel genetic and environmental factors.

We first analyzed FA for the mandible as a whole, then repeated the analysis for each partition of the jaw, superimposed separately. The first analysis places each part within the context of the mandible as a whole, the second examines correlations among parts, taken individually. We partitioned the mandible into 11 parts, each of which is a complex, multidimensional trait, a matrix of coordinates rather than a single dimension (Monteiro et al. 2005). Some of the partitions subdivide putative modules into two or more parts (e.g., the partitions of the coronoid and condyloid into proximal and distal parts). This partitioning of putative modules allows us to examine correlations within as well as between hypothesized modules. The partitions are named according to their position relative to the teeth; i.e., the proximal part of the condyloid is the one nearest the teeth.

The two sets of partitions shown in Fig. 3 differ in where they place the boundary between angular process and ramus, resulting in different subdivisions of the region on which the superficial masseter inserts. The first set of partitions divides the insertion site of the superficial masseter into angular and ramal components, so one partition corresponds to the angular process, the other to the ramus (Fig. 3A). The other set of partitions keeps the insertion site intact, extending this partition into the ramus, which leaves only landmark 13 to mark the ventral border of the ramus (Fig. 3B). We use these two sets of partitions because the angular process and ramus originate within different mesenchymal condensations that contain different cell populations, but the same muscle inserts on both partitions. Thus, according to hypotheses that emphasize the impact of cellular origin on modularity, the angular process is independent of the ramus even though the same muscle inserts on both, but according to hypotheses that emphasize the role of muscle-bone interactions on modularity, the angular process should be correlated with the ramus. For brevity, we refer to the first set of partitions as the "Angular" set and the second as 


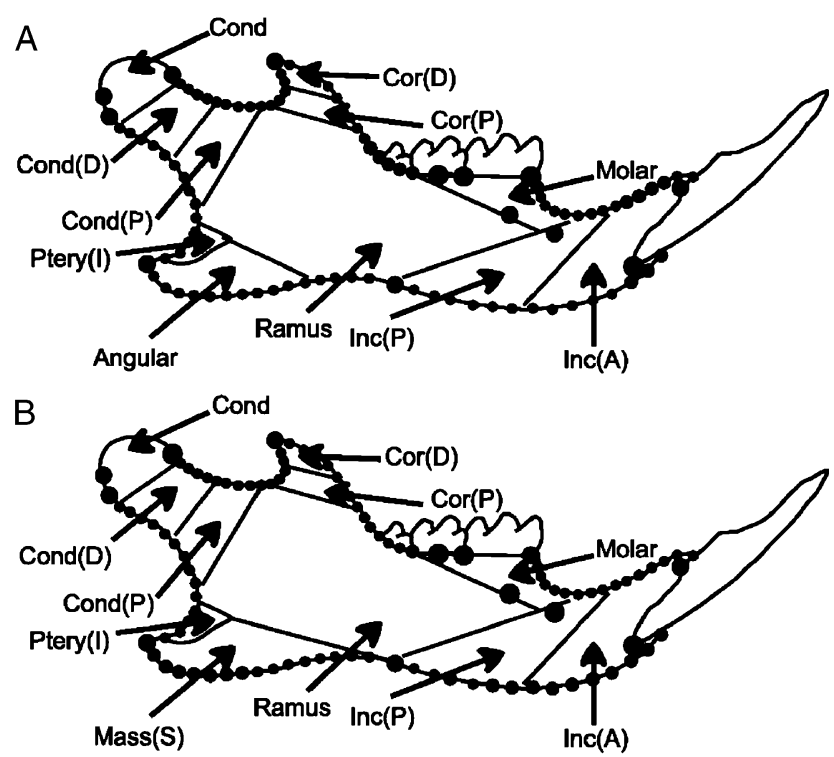

Fig. 3. Partitions of the mandible. The two sets of partitions differ in the boundary between angular process and ramus. (A) "Angular partitions"; the insertion site of the superficial masseter is divided into angular and ramal components, with the angular partition corresponding to the angular process, the ramal partition to the ramus. (B) "Superficial masseter partitions"; the insertion site of the superficial masseter is kept intact within the partition, extending its boundary onto the ramus, leaving only landmark 13 to mark the ventral border of the ramus.

the "Superficial masseter" set. One landmark (\#4), located on the anterior of the masseteric ridge, was not included in any partition because it is far from all other landmarks allocated to the ramus and because variation in the position of the ridge on the ramus could be confounded with variation in proportions of the ramus.

The FA and symmetric (individual) components of variation obtained for each partition, analyzed separately, were used to estimate correlations among partitions following the method devised by Monteiro et al. (2005). That method involves calculating matrices of pairwise Procrustes distances between all individuals for each partition. Then matrix correlations are computed between the distance matrices. The rationale for this procedure is that the matrices of pairwise Procrustes distances for each partition contain all the information about the structure of variation within shape space, so correlations are estimated between whole shapes not between individual landmarks. The correlation between distance matrices tells us whether variation in the shape of one partition is associated with variation in the shape of another. When both partitions show the same pattern of shape differentiation among individuals, the two matrices should be closely associated, yielding a statistically significant matrix correlation. Conversely, when variation in one partition is not associated with variation in another, the two will differ in their structures of variation, and the distances will not be associated, yielding a matrix correlation near zero. Calculation of the pairwise Procrustes distances within partitions, and correlations between distance matrices was done in Coriandis (Marquez 2007a).
Testing a priori hypotheses of modularity and integration We test five a priori hypotheses by comparing the observed correlation matrices to the matrices of expected correlations ("target matrices"). Following the method used by Cheverud et al. (1991) in their analysis of mandibular integration, target matrices are constructed by placing a one where the hypothesis predicts that traits are integrated and a zero where the hypothesis predicts that traits are independent. When the hypothesis predicts a hierarchical structure of integration, correlations will be intermediate between zero and one.

The first hypothesis ("Condensation") predicts that structures derived from the same mesenchymal condensation will be integrated with each other and independent of structures derived from different condensations. This condensation hypothesis predicts that parts of a single mandibular process are correlated with each other, as are parts of the incisor alveolus, but the processes are not correlated with each other, nor with the ramus or dental alveoli and the ramus and alveoli are uncorrelated with each other.

The second hypothesis ("Developmental History") predicts that parts arising from condensations populated by the same cell types will be integrated. The two dental alveoli will therefore be correlated because the cells within both will differentiate to form odontoblasts and osteoblasts. Similarly, the three mandibular processes will be correlated because they all arise from cell populations that differentiate to form osteoblasts and secondary chondroblasts. The ramus is a discrete module, arising from a cell population that differentiates to form osteoblasts and, when needed for repair, primary chondroblasts. Because the coronoid process originates within the mass of the temporalis muscle, it is predicted to be less tightly correlated with the condyloid and angular processes than they are with each other (Atchley et al. 1985; Atchley and Hall 1991; Cheverud et al. 1991). Thus, this hypothesis predicts three levels of integration. The highest level (represented by 1.00 in the target matrix) is expected for the two alveoli and partitions of the same process or alveolus. The intermediate value $(0.67$ in the target matrix) is expected for parts of the angular and condyloid process, and the third level ( 0.33 in the target matrix) is expected for correlations between coronoid partitions and those of the other two processes. All other combinations of partitions are expected to be uncorrelated ( 0.0 in the target matrix).

The remaining hypotheses all explain mandibular integration in terms of muscle-bone interactions but they make different predictions regarding the spatial extent and location of those interactions. According to the third, "Muscle," hypothesis, each mandibular process is an integrated unit, independent of the others because each is affected by a different muscle. The ramus and angular, however, are integrated because both are affected by the masseter (Cheverud et al. 1991). The alveoli also form an integrated unit because both are affected by teeth. In the target matrix used to test this hypothesis, values of 1.0 represent expected correlations between (1) parts of a single mandibular process, (2) the ramus and two angular partitions, and (3) molar alveolus and partitions of the incisor alveolus. Values of 0.0 represent expected correlations between (1) different mandibular processes, (2) ramus and mandibular processes other than the angular and (3) dental alveoli and other parts of the jaw.

The fourth hypothesis ("Insertion-Sites") postulates a more localized impact of muscles on the mandible in that integration is 
expected solely where an individual muscle inserts. Rather than postulating that muscle-loading can affect a whole process, this model proposes that muscles affect only the local region where they insert. Thus, a single mandibular jaw process will comprise more than one module when more than one muscle inserts on it, as in the case of the angular on which both the internal pterygoid and superficial masseter insert. This model also predicts that two mandibular processes could comprise a single module should the same muscle insert on both (Badyaev et al. 2000; Badyaev and Foresman 2004; Badyaev et al. 2005). This hypothesis makes no predictions about integration elsewhere, thus, interpreted strictly, there are no predictions regarding dental alveoli or regions along the mandibular processes where no muscles insert. We cannot test that strict formulation of the hypothesis because our partitions typically span more than a single muscle insertion site. Had we partitioned both coronoid and condyloid so that each partition contained a single insertion site, much of the jaw would not lie within any partition and only information about local curvature would be contained in the data. Thus, our formulation of the hypothesis does not strictly follow that of Badyaev and colleagues. Instead, we predict that parts on which the same muscle inserts will be correlated even if the part spans more than a single insertion site. Given our partitioning of the mandible, the target matrix for this hypothesis contains values of 1.0 for correlations between (1) proximal and distal coronoid, (2) proximal and distal condyloid (excluding the condyle) and (3) between all three partitions of the alveoli, and 0.0 for all other combinations such as the parts of the angular on which the external pterygoid inserts and that on which the superficial masseter inserts. Two versions of this model are needed because part of the insertion site of the superficial masseter is contained within the ramal partition of the "Angular" set, whereas the entire insertion site is contained within the superficial masseter partition of the "Superficial Masseter" set. Thus, one version of this model Insertion-Sites(A) predicts a correlation of 0.25 between ramal and angular/superficial masseter partitions, and the other, InsertionSites(B) predicts a correlation of 0.0 between those partitions.

The fifth hypothesis ("Front/Back") predicts that the entire muscle-bearing region forms one module, whereas the toothbearing region forms a second, a currently favored hypothesis of mandibular modularity (e.g., Mezey et al. 2000; Klingenberg et al. 2003; Cheverud 2004; Jojic et al. 2007). Both developmental and functional rationales have been offered for this model, both of which are succinctly stated by Cheverud (1996). The developmental rationale is that no known physiological or developmental process links dental to muscle development and the functional rationale is that the mandible contains two functionally independent parts: one serves to bear muscles, the other teeth. To test this hypothesis, values of 1.0 represent expected correlations between (1) all three mandibular processes and ramus, and (2) the three alveolar partitions. Values of 0.0 represent expected correlations between the three alveolar partitions and the ramus plus mandibular processes.

The statistical significance of the correlation between observed and target matrices is assessed by a Mantel test, which randomly permutes rows and columns, yielding the distribution of matrix correlations for unrelated matrices (Dietz 1983). To determine if one hypothesis fits better than another, we used the procedure outlined by Dow and Cheverud (1985); according to this
Table 1. Correlations between target matrices encoding the a priori models of mandibular modularity; Correlations are given above the diagonal, $\boldsymbol{P}$-values based on Mantel tests below

\begin{tabular}{|c|c|c|c|c|c|}
\hline & $\begin{array}{l}\text { Conden- } \\
\text { sation }\end{array}$ & $\begin{array}{c}\text { Developmental } \\
\text { history }\end{array}$ & Muscles & $\begin{array}{l}\text { Insertion- } \\
\text { sites }\end{array}$ & $\begin{array}{c}\text { Front } \\
\text { back }\end{array}$ \\
\hline Condensation & & 0.598 & 0.568 & 0.449 & 0.336 \\
\hline $\begin{array}{l}\text { Developmental } \\
\text { history }\end{array}$ & $<0.001$ & - & 0.597 & 0.664 & 0.664 \\
\hline Muscles & $<0.001$ & 0.004 & - & 0.715 & 0.398 \\
\hline Insertion-sites & 0.005 & $<0.001$ & 0.001 & - & 0.278 \\
\hline Front/back & 0.019 & 0.006 & 0.002 & 0.080 & - \\
\hline
\end{tabular}

procedure, the target matrices are first standardized to a mean of zero and variance of one, then one target matrix is subtracted from the other. This difference matrix is then compared with the observed correlation matrix by a standard Mantel test. Should the correlation be statistically significant and positive, the observations fit the predictions of the first hypothesis better than those of the second; alternatively, a significant and negative correlation means that the second hypothesis fits significantly better than the first. Mantel tests were done in PopTools (Hood 2008).

Our ability to evaluate the relative fit of these five hypotheses is limited by the similarity among them (Table 1). Nearly all target matrices are significantly similar to each other, the only exception being the two models predicting the greatest and least integration, the Front/Back and Insertion-Sites models. Nevertheless, there are critical differences between all hypotheses, especially regarding the angular process. The angular process is predicted to be (1) integrated and independent of all other jaw components (Condensation model), (2) integrated and correlated only with the other mandibular processes, especially with the condyloid (Developmental History model), (3) integrated and correlated with the other processes and with the ramus (Front/Back model), (4) integrated and correlated only with the ramus (Muscle model), or (5) not integrated and not correlated with any other part except when the superficial masseter insertion overlaps the ramus (Insertion-Sites model). Such specific but subtle differences among models are best isolated using exploratory methods, which can also discern correlations not anticipated by any models.

\section{Exploratory analysis of modularity and integration}

We use two approaches to clustering, one that is a strict hierarchical method and one that relaxes the assumptions of hierarchical clustering and allows for associations between traits belonging to different clusters. A defining characteristic of standard hierarchical clustering methods is that they are ultrametric, meaning that all traits are placed equally distant from the root and every distance within a cluster is smaller than every distance to a trait outside that cluster. We use Ward's method (1963) of hierarchical clustering, one of the most widely used in studies of morphological integration (e.g., Cheverud 1982; Goswami 2006; Willmore et al. 2006), because it aims to produce compact, spherical clusters by minimizing the variance of intra-cluster distances. 
Cluster analyses will find clusters even if the data show little evidence of hierarchical structure making it important to assess the fit of the data to the dendrograms representing clusters inferred from the analysis. That fit is usually assessed by the cophenetic correlation (Sneath and Sokal 1973), which measures the correlation between the observed correlation matrix (transformed to a distance matrix by subtracting each correlation from 1.0) and the distances between objects produced by the clustering method. Values lower than 0.85 are usually taken as evidence of distortion. Ward's method, however, does not maximize the cophenetic correlation and can therefore yield low values, especially when compared with UPGMA, the clustering method that yields the highest cophenetic correlation (Farris 1969). An alternative metric more suited to Ward's method is the agglomeration coefficient (AC), which measures the hierarchical structure of the data (Kaufman and Rousseeuw 1990). The AC is calculated from the distance between each observation and the first cluster with which it is merged, divided by the dissimilarity between the last-merged objects. Denoting that ratio as $m_{i}$, and calculating the AC for $n$ traits,

$$
\mathrm{AC}=\sum_{1}^{n-1}\left(1-m_{i}\right)
$$

The AC, which ranges from 0 to 1 , is high when the first merged traits are far more similar to each other than the last merged objects. Hierarchical clustering was done in Systat (Wilkinson 2000), calculation of the cophenetic correlation and AC were done in the "cluster" package (Maechler et al. 2005) in R (R Development Core Team 2008)

Correlation structure can also be explored by relaxing the requirement that all distances within a cluster be smaller than distances to traits outside the cluster and the requirement that all traits outside the cluster be equally far from all traits within (Sattath and Tversky 1977). Trees produced this way are additive, not ultrametric, but this approach still makes the crucial assumption that every trait belongs to only one cluster. That assumption can be relaxed by allowing for reticulations among clusters in the network, a method used to investigate horizontal gene transfer and hybridization among taxa (Makarenkov and Legendre 2004; Makarenkov et al. 2004).

To determine whether reticulations are demanded by data, a least-squares approach is used to minimize the squared deviations between the original dissimilarities and the associated reticulation distances. Reticulations are added to the additive tree, minimizing:

$$
Q_{1}=\frac{Q(N)^{1 / 2}}{\left(\frac{n(n-1)}{2-N}\right)}
$$

where $Q(N)$ is the sum of the squared deviations between the original distances and the reticulation distances, $n$ is the number of objects (traits) and $N$ is the number of edges in the graph. If adding reticulations does not improve significantly upon the additive tree, none are added. $Q_{1}$ is a relatively conservative criterion, adding fewer reticulations than an alternative $\left(Q_{2}\right)$ that has the leastsquares criterion in the numerator. Reticulated networks were analyzed in T-Rex (Makarenkov 2000), using the additive tree option for the tree construction and $Q_{1}$ as the optimality criterion.

\section{RESULTS}

\section{Sources and dimensions of variation}

Variation among individuals is clearly the dominant source of phenotypic variation in jaw shape (Table 2). Directional asymmetry explains little variation although the effect is statistically significant. FA is statistically significant even after among-family variation is removed from the data $(P<0.001)$, but then its contribution to variation decreases to $7.8 \%$.

Symmetric variation among individuals is fairly well concentrated in shape space. The first three principal components account for $57 \%$ of the symmetric variation in these 107 dimensional data (Fig. 4). Similarly, the first four principal components account for $46 \%$ of variation in FA after variation among families is removed from that component (Fig. 4). Variation in width and orientation of the angular, condyloid, and coronoid dominate the first three principal components of symmetric shape and the first four components of FA. However, the patterns of variation differ between the principal components of these two data sets even though the two matrices are significantly similar; the correlation between the symmetric and FA covariance matrices is $0.67(P<0.001)$. In particular, in the symmetric data, the entire coronoid is primarily associated with variation in the condyloid (PC1), with the more distal coronoid varying in association with either the region where the superficial masseter (PC2) or internal pterygoid insert (PC3). In contrast, FA spanning the coronoid is associated with the insertion of the superficial masseter ( $\mathrm{PC} 1$ ), and that of the more proximal coronoid is associated with the distal condyloid. FA in the region where the external and internal pterygoids insert is associated with the region where the superficial masseter extends onto the ramus (PC4).

\section{Testing a priori hypotheses of integration/ modularity}

Two a priori models, the Condensation and Muscle models, predict the structure of correlations based on the symmetric component of variation for both sets of partitions moderately

Table 2. Procrustes analysis of variance of shape explained by variation among individuals, differences between sides and the interaction between the two main factors

\begin{tabular}{lcccccc}
\hline Effect & SS & df & MS & F & $P$ & \% Total \\
\hline Individual & 0.1712 & 9.900 & $2.48646 \mathrm{E}-06$ & 8.43 & $<0.001$ & 87.83 \\
Sides & 0.0015 & 110 & $4.42865 \mathrm{E}-06$ & 6.57 & $<0.001$ & 0.77 \\
Individual $\times$ & 0.0203 & 9.900 & $4.93854 \mathrm{E}-07$ & 7.75 & $<0.001$ & 10.41 \\
$\begin{array}{l}\text { side } \\
\begin{array}{l}\text { Measure- } \\
\text { ment error }\end{array}\end{array}$ & 0.0053 & 20,020 & $8.70833 \mathrm{E}-08$ & & & 2.72 \\
\hline
\end{tabular}



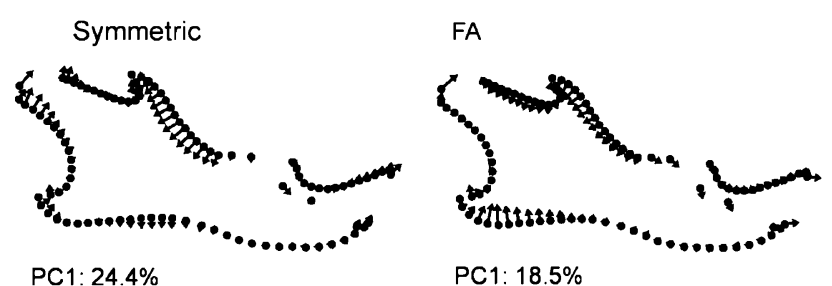

PC1: $18.5 \%$

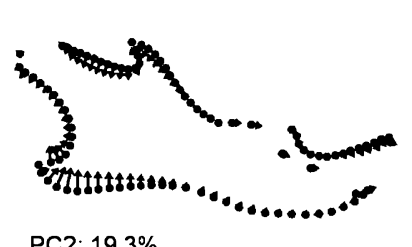

PC2: $19.3 \%$

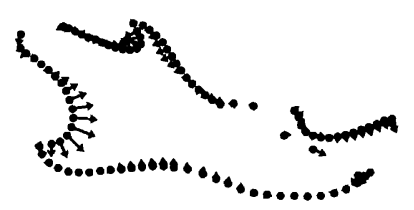

PC3: $13.2 \%$
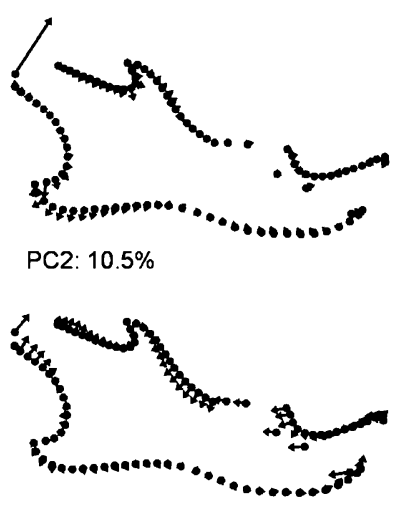

PC3: $9.0 \%$

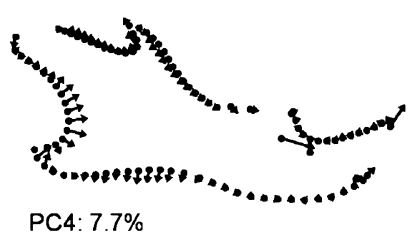

Fig. 4. Principal components of symmetric variation and FA for the mandible as a whole.

well (Table 3); a third, the Insertion-Sites model, is just marginally nonsignificant or significant depending on partitions and version of the model. The Condensation and Muscle models fit equally well $(P>0.50$ for the difference-matrix fitted to both sets of partitions). Models fitted to the correlation structure of FA provide equally equivocal results; the Condensation, Muscle and Insertion-Sites models (both versions) are all significantly correlated with the data and none fits significantly better than the others $(P>0.15$ for all difference-matrices contrasting these three hypotheses, fitted to both sets of partitions). The results from these tests of a priori models do not provide compelling support for the hypothesis that direct epigenetic interactions between muscle and bone integrate the mandible because even the best-fitting hypothesis of muscle-bone interactions fails to improve upon the Condensation hypothesis.

\section{Exploratory analysis}

The cophenetic correlations and ACs are all low, regardless of the data set and clustering method (Table 4). All cophenetic correlations are below 0.85 , indicating that the dendrograms representing the hierarchical structure in the data distort the

Table 3. Correlations between a priori hypotheses and the correlation matrices among partitions calculated from the individual and FA components of variation

\begin{tabular}{lcccccc}
\hline & \multicolumn{2}{c}{ Angular } & & \multicolumn{2}{c}{$\begin{array}{c}\text { Superficial } \\
\text { Masseter }\end{array}$} \\
\cline { 2 - 3 } \cline { 5 - 6 } $\begin{array}{l}\text { Model } \\
\text { Symmetric }\end{array}$ & $\mathrm{R}$ & $P$ & & $\mathrm{R}$ & $P$ \\
\hline Condensations & 0.448 & 0.003 & & 0.430 & 0.003 \\
Developmental history & 0.150 & 0.133 & & 0.132 & 0.178 \\
Muscles & 0.397 & 0.001 & & 0.334 & 0.008 \\
Insertion-sites(A) & 0.249 & 0.044 & & 0.238 & 0.067 \\
Insertion-sites(B) & 0.241 & 0.052 & & 0.240 & 0.053 \\
Front/back & 0.220 & 0.130 & & 0.237 & 0.100 \\
FA & & & & & \\
Condensations & 0.244 & 0.026 & & 0.234 & 0.029 \\
Developmental history & 0.182 & 0.136 & & 0.216 & 0.078 \\
Muscles & 0.290 & 0.008 & & 0.268 & 0.001 \\
Insertion-sites(A) & 0.377 & 0.007 & & 0.322 & 0.007 \\
Insertion-sites(B) & 0.356 & 0.007 & & 0.361 & 0.002 \\
Front/back & 0.161 & 0.210 & 0.200 & 0.210 \\
\hline
\end{tabular}

The partitions labeled "Angular" correspond to those shown in Fig. 3A, those labeled "Superficial Masseter" correspond to the partitions shown in Fig. 3B. $\mathrm{R}=$ matrix correlation between observed and target matrix, $P=P$-value based on the Mantel test.

observed correlation matrix. The ACs are also very low, indicating that the data are not strongly hierarchical and are therefore not suitable for analysis by hierarchical methods. Relaxing the requirements imposed by hierarchical clustering methods reveals the weakly hierarchical and complex

Table 4. Cophenetic correlations $\left(R_{\mathrm{C}}\right)$ and agglomeration coefficients $(\mathrm{AC})$ for dendrograms derived from the correlation matrices among partitions calculated from

the symmetric and FA components of variation

\begin{tabular}{lccccc}
\hline & \multicolumn{2}{c}{ Angular } & & \multicolumn{2}{c}{ Superficial Masseter } \\
\cline { 2 - 3 } & $R_{\mathrm{C}}$ & $\mathrm{AC}$ & & $R_{\mathrm{C}}$ & $\mathrm{AC}$ \\
\hline Symmetric & & & & \\
Wards & 0.688 & 0.336 & & 0.669 & 0.364 \\
UPGMA & 0.786 & 0.194 & & 0.811 & 0.206 \\
FA & & & & \\
Wards & 0.686 & 0.298 & & 0.634 & 0.300 \\
UPGMA & 0.778 & 0.228 & & 0.783 & 0.215 \\
\hline
\end{tabular}

The set of partitions labeled "Angular" corresponds to the partition shown in Fig. 3A, which has the anterior region of the insertion of the superficial masseter allocated to the ramus partition; the set of partitions labeled "Superficial Masseter" corresponds to the partitions shown in Fig. 3B, which has the boundary between superficial masseter and ramus partitions shifted anteriorly so that the insertion of that muscle lies entirely within the superficial masseter partition. 


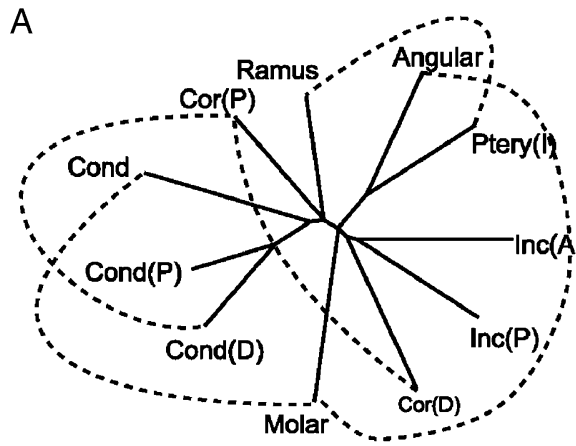

C

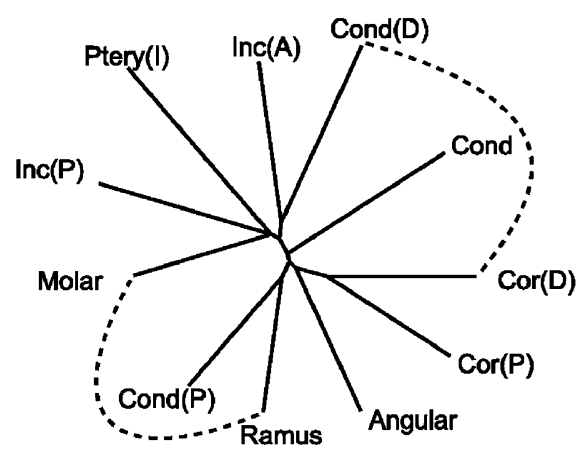

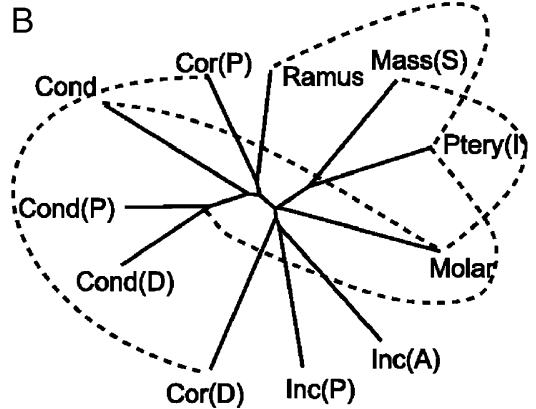

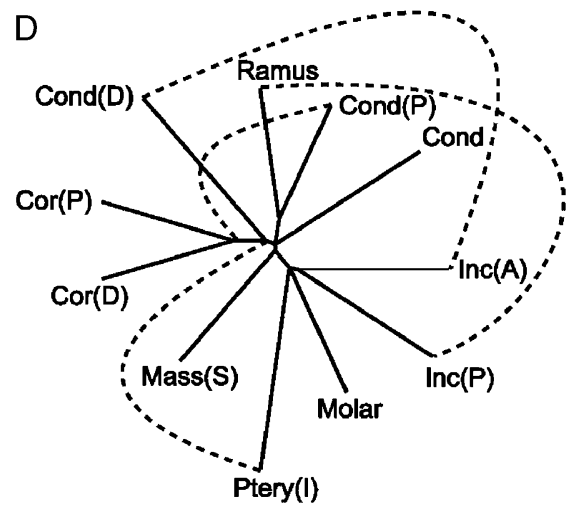

Fig. 5. Reticulating networks. (A) Symmetric variation "Angular partition," (as shown in Fig. 3A); (B) Symmetric variation "Superficial Masseter partition" (as shown in Fig. 3B); (C) FA "Angular partition" (as shown in Fig. 3A); (D) FA "Superficial Masseter partition" (as shown in Fig. 3B). structure of mandibular integration (Fig. 5). The typically small internal branches, which differ little in length, plus the typically long external ones, show that mandibular integration is not characterized by a nested hierarchy of tightly correlated traits.

The branching patterns for symmetric variation are very similar despite differences in the partitioning of the angular process (Fig. 5, A and B). In both cases, the parts of the mandible are divided into two main clusters: (1) ramus, proximal coronoid and condyloid process, and (2) dental alveoli, parts of the angular process and distal coronoid process. The first main cluster is divided into two subgroups, one containing the ramus and proximal coronoid, the other containing the parts of the condyloid. The second main cluster is divided into slightly different subgroups depending on the boundary between superficial masseter and ramus. In both cases, one group includes the internal pterygoid insertion plus the other part of the angular ("angular" or "superficial masseter"), and a group that includes the two incisor partitions plus distal coronoid. The difference lies in the links between the molar alveolus and the other parts. When part of the superficial masseter insertion is partly contained within the ramus, the molar is linked to incisor plus coronoid group (Fig. 5A) whereas when the superficial masseter insertion site is contained wholly in the superficial master partition, the molar is linked to the internal pterygoid-superficial masseter group (Fig. 5B). The reticulations, like the main branching patterns, are similar between the two sets of partitions. Reticulations linking parts of the condyloid process to the molar and prox- imal coronoid are found in both analyses as is a reticulation linking the molar to angular/superficial masseter partitions. The difference lies in a reticulation that links proximal to distal coronoid in (Fig. 5A) or one that links the condyloid process to the internal pterygoid insertion (Fig. 5B). Regardless of some differences in details, the structure of integration revealed by these trees contradicts the basic premise of all a priori hypotheses - that the dental alveoli are independent of muscle-bearing structures.

The patterns of integration seen in FA are similar to those of symmetric variation in that there is a group including the incisor and molar but in analyses based on FA, the internal pterygoid insertion is included within this group of partitions (Fig. 5, C and D). Depending on the location of the boundary between angular and ramus, the distal condyloid partition may be included within that group, and the incisor partitions may form a subgroup. The second major branch unites the two coronoid partitions, which are always paired in analyses based on FA, plus the ramus, which is always paired with the proximal partition of the condyloid process. Further resolution depends on extremely short branches that are sensitive to the boundary between angular and ramus, which also determines whether the distal condyloid partition is included in this group. Despite the instability, there are only two reticulations linking parts belonging to different groups in the analyses that allocate part of the superficial masseter insertion to the ramus (Fig. 5C). One of these links the molar to the ramus partitions, the other links distal parts of the coronoid and condyloid 
processes. Neither of those connections appears when the superficial masseter insertion site is contained wholly in the "superficial masseter" partition (Fig. 5D). Under that condition, there is a link between the proximal condyloid and coronoid process. In addition, reticulations link the incisor to the ramus or distal condyloid and another links the internal pterygoid insertion to the group containing coronoid and distal condyloid. The greater number of very short branches within the major groups, and the greater number of reticulations that bridge the groups, again indicates that the clusters are weakly supported and sensitive to the delimitation of the angular from ramus partitions. As seen in the trees representing the correlation structure of symmetric variation, the dental alveoli are not independent of muscle-bearing structures.

The spatial structure and complexity of relationships among traits may be most evident when correlations are displayed on the mandible (Fig. 6). Only those exceeding 0.24 are shown because these are at least one standard deviation higher than the average of 0.16 and are typically statistically significant even if $P$-values are adjusted for the 55 statistical tests. Because this diagram shows far less of the structure of integration than revealed by the networks, the pattern is less complex but even relatively high correlations show a network structure. In both the "Angular" and "Superficial Masseter" data sets, there is a network of relationships among ramus, condyloid and proximal coronoid, with ramus also linked to the internal pterygoid insertion (Fig. 6, A and B).

The FA correlations show an even more complex network of associations, especially between the ramus and the other parts of the mandible (Fig. 6, C and D). The ramus appears to be integrated with other parts that are otherwise unrelated to each other. When the superficial masseter insertion is allocated both to the angular and ramus (Fig. 6C), there is no integration between the angular and other mandibular processes; in contrast, when the superficial masseter is contained solely within the angular process, the insertion site of that muscle is correlated with the proximal condyloid (Fig. 6D) and the correlation between that insertion site and the molar increases but that between molar and ramus decreases. The superficial masseter-condyloid and ramus-molar versus ramus-superficial masseter correlations thus appear to depend on the boundary between ramus and angular process. When landmarks within the ramal portion are allocated to the superficial masseter and therefore excluded from the ramus, they augment the superficial masseter-condyloid and superficial-masseter-molar association, whereas including them within the ramus augments the ramus-molar association.

\section{DISCUSSION}

Our analysis of mandibular integration reveals an unanticipated complexity of relationships among parts. Rather than
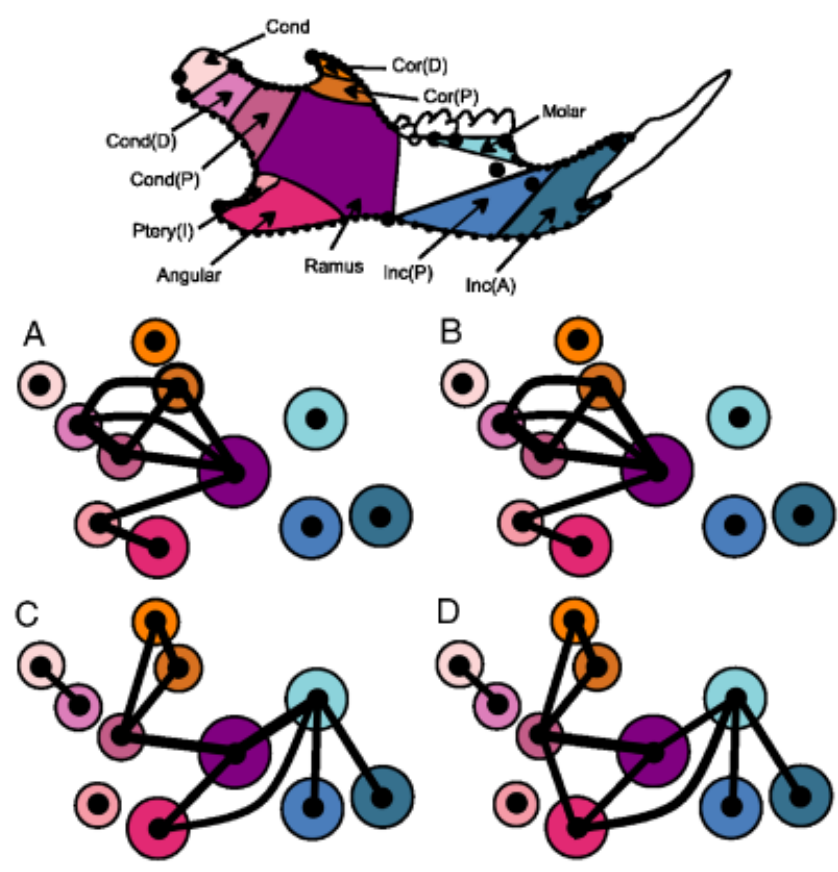

Fig. 6. Correlations exceeding $r=0.24$ drawn on a schematic of the jaw in which circles represent centroids of partitions. Line weights of the edges connecting partitions are proportional to the correlation coefficient. (A) Symmetric variation "Angular partition" (as shown in Fig. 3A); (B) Symmetric variation "Superficial Masseter partition" (as shown in Fig. 3B); (C) FA "Angular partition (as shown in Fig. 3A); (D) FA "Superficial Masseter partition" (as shown in Fig. 3B).

finding modules of mutually intercorrelated parts independent of others, we instead find that some parts, such as the ramus, are highly associated with other parts that are not otherwise integrated with each other. We also find that partitions of some developmental modules are more highly associated with parts of other modules than with other parts of their own module. That complexity is not predicted by any of the developmental models, not even by the ones significantly correlated with the data. Even those models that fit significantly do not fit the data well - no correlation between models and data surpasses 0.45 (Table 3). Such weak correlations are not surprising because the models are admittedly crude, and weak correlations are frequently found in studies using the approach to model construction taken herein (e.g., Cheverud et al. 1991; Cheverud 1995; Hallgrímsson et al. 2004). Yet, the crudity of the models is not the only or even the most important cause of their poor fit. The three more important reasons are: (1) no model predicts the complex networks of interactions discerned by our exploratory analyses, (2) no model predicts correlations between the proximal parts of the coronoid and condyloid, which can exceed those between the proximal and distal parts of the same process and (3) no model predicts the correlation between tooth- and muscle-bearing units. 
The developmental models tested herein all anticipate a simple structure of modularity. Most predict that subsets of traits derived from the same developmental module are highly intercorrelated with each other and (nearly) independent of other developmental modules. The only other structures anticipated by these modules is a hierarchical pattern of modules nested within larger units or correlations limited to parts on which the same muscle inserts. Our data do not show any of those structures. Rather we find a network of partially overlapping integrated units, with parts of some modules integrated with parts of other developmental modules, such as the proximal parts of condyloid and coronoid integrated with each other, rather than with the other parts of their own developmental module (Figs. 5 and 6). Additionally, some parts, most notably the ramus, are integrated with structures that are not mutually correlated with each other, such as the region of the angular process on which the superficial masseter inserts and the distal part of the condyloid process. Partial overlap is consistent with the general theory of modularity (Wagner and Altenberg 1996; Magwene 2001; Hallgrímsson et al. 2007a, b), but our results show such a high degree of overlap that the mandible as a whole could be considered one single weakly integrated module or else it could be regarded as not modular at all.

Such a complex network of interactions is not anticipated by any of the currently favored developmental models tested in this study. A complex and nearly unpredictable structure of integration is, however, predicted by the palimpsest model (Hallgrímsson et al. 2007a, b). According to that palimpsest model, the structure of (co)variation results from the cumulative effect of multiple processes that generate (co)variance, integrated over time. Because these processes differ in their spatial structure and extent, they can amplify or dampen the variation produced by individual (co)variance-generating processes. The accumulated effects of many such processes acting over time, and over the space of the mandible, could explain the complexity revealed in our results. A complex, yet spatially organized pattern is especially likely to result from the net effects of spatially distributed physical forces, such as those due to mechanical factors, integrated over the entire mandible.

Several of the strongest correlations found in our data are not anticipated by any of the developmental models tested herein. The details of these correlations differ between the symmetric and FA components of variation, as well as between the two sets of partitions. However, one recurrent pattern is that the proximal parts of the coronoid and condyloid processes are more highly integrated with each other and with the ramus than they are with the distal parts of the same process. Another particularly interesting correlation, although not invariably one of the highest, is the correlation between molar alveolus and ramus and sometimes also angular process. This correlation is among the highest found in the structure of FA. We are not the first to detect a correlation containing the molar and ramus (see, e.g., Atchley et al. 1992; Atchley 1993; Monteiro et al. 2005). That correlation is also implied by the relatively high trace correlation between front and back of the jaw (Klingenberg et al. 2003). Interestingly, this correlation is found not only in studies of the shapes of partitions (Klingenberg et al. 2003; Monteiro et al., 2005, this study), but also in their relative sizes (Atchley et al. 1992; Atchley 1993), which suggests that the mechanisms integrating growth act differentially across each part, stimulating growth more in some directions than others. The correlation between ramus and molar alveolus has been found in morphologically diverse and distantly related rodents, including Old World myomorphs (laboratory rats and mice, Atchley 1993; Atchley et al. 1993), in a natural population of house mice, as well as in our laboratory population of a New World myomorph, and in a natural population of a distant relative of myomorphs, the hystricomorph Trinomys gratiosus (Monteiro et al. 2005). This correlation, despite being repeatedly found by empirical studies, is not predicted by available developmental models. All those models are predicated on the assumption that muscle-bearing and tooth-bearing parts are developmentally and functionally independent units.

Considerations of the mechanics of mastication and biting have suggested an alternative view of mandibular functional integration, one that does predict a correlation between teethand muscle-bearing parts of the jaw (Zelditch and Carmichael 1989; Monteiro et al. 2005). The fact that this correlation is found in the FA component suggests that it arises from direct epigenetic interactions and therefore ought to be predictable from developmental theory. That it is found in the FA component also suggests that this may be one of those correlations between functionally coupled traits that is built into the developmental system. That none of the available developmental models predicts this correlation may be due to the models deriving their predictions from what is known about embryonic muscle-bone interactions. Embryonic loading apparently has highly local effects, evident in the effects of mutations that reduce or eliminate embryonic muscle contractions (Hall and Herring 1990; Herring 1993; Rot-Nikcevic et al. 2006, 2007). Those mutations tend to disproportionately affect the coronoid and angular processes; the coronoid is entirely eliminated, the angular is severely reduced, and the condyloid is greatly narrowed and elsewhere the anomalies are relatively modest (Rot-Nikcevic et al., 2006, 2007). But muscle-bone interactions continue throughout life and embryonic muscle-loading differs strikingly from that occurring later during mastication and biting. A critical difference between embryonic muscle-bone interactions and those occurring due to functioning jaws is that in mastication and biting there is resistance at the teeth.

None of the available developmental models consider the long-term effects of jaw function, but the biomechanics of mastication and biting are relevant not only for predicting 
patterns of functional coupling but also for modeling morphogenetic processes. As Herring (1993) points out, function is epigenetic. She characterizes the role of function in cranial development as being to produce mechanical loads, which deform bone, and that deformation (measured by strain) is the epigenetic signal stimulating bone modeling (Carter et al. 1996; Burr et al. 2002; Harris et al. 2004; Henderson et al. 2004). In the presence of resistance at the teeth, we should anticipate correlations due to coupling between forces generated by muscle contraction and those experienced at teeth. We might therefore anticipate finding correlations due to that coupling, and we now have sufficient empirical grounds to expect such a correlation. However, we still have no realistic developmental theory that can predict the correlations over the jaw. Such a theory may be necessary to determine whether muscle-bone interactions are actually responsible for the correlations repeatedly found between ramus and alveoli, because other signaling interactions might also explain correlations between these units; we cannot simply assume that they arise from the coupling between forces of contraction and occlusion.

It is reasonable to expect that masticatory strain is one of the signals contributing to FA because the spatial structure of strain is unlikely to be perfectly symmetrical, and that structure is likely to vary among individuals. We might therefore anticipate spatially organized FA due to masticatory strains. However, we do not presently know how strain is distributed across any rodent mandible and, absent that knowledge, we cannot predict how variation in its distribution would induce correlations among parts of the jaw. We might expect that integration caused by the spatial distribution of bone strain would show a spatially organized pattern, such as found in this study, but bone-strain is not the sole spatially organized developmental signal. To isolate this variation in strain as the cause of integration we need precise quantitative models of its spatial distribution so that we can test the model as a whole rather than pick out predictions potentially consistent with that hypothesis. The correlations between proximal condyloid and coronoid also need a developmental explanation, and a useful theory should also predict when correlations might arise between incisors and ramus rather than molars and ramus. Our exploratory analyses have isolated relationships among parts not anticipated by any current developmental models, but exploratory methods are not a satisfying alternative to a predictive theory.

Our results argue for more a complex epigenetic theory of developmental integration than supposed by current models, but until we understand the spatial distribution of muscle loading and its resultant strains we cannot devise those more complex models. Our inability to devise those models also makes it difficult to predict how readily integration caused by direct epigenetic interactions should evolve. Those direct interactions may be highly conservative because they are intrinsic to the organization of developmental systems, but strains engendered by jaw function might be no more historically conservative than the spatial distribution of bone strain is. To devise a realistic theory of the causes of direct epigenetic interactions, which can predict both the structure and evolutionary dynamics of modularity, we likely will need principles rooted in the biomechanics of development as much as in developmental genetics.

\section{Acknowledgments}

We thank P. Myers of the University of Michigan, Museum of Zoology Mammal Division for access to the specimens, P. Hartley for assistance with photography, and A. Dengate for assistance with digitizing. We also gratefully acknowledge the insightful comments of B. Hall, C. Ross and two anonymous reviewers.

\section{REFERENCES}

Adams, D. C., Rohlf, F. J., and Slice, D. E. 2004. Geometric morphometrics: ten years of progress following the 'revolution.' Ital. J. Zool. 71: 5-16.

Andresen, P. R., Bookstein, F. L., Conradsen, K., Ersbøll, B., Marsh, J., and Kreiborg, S. 2000. Surface-bounded growth modeling applied to human mandibles. IEEE Trans. Med. Imag. 19: 1053-1063.

Atchley, W. R. 1983. A genetic analysis of the mandible and maxilla in the rat. J. Craniofac. Genet. Dev. Biol. 3: 409-422.

Atchley, W. R. (1993). Genetic and developmental aspects of variability in the mammalian mandible. In J. Hanken and B. K. Hall (eds.). The Skull. University of Chicago Press, Chicago, pp. 207-247.

Atchley, W. R., Cowley, D. E., Vogl, C., and McLellan, T. 1992. Evolutionary divergence, shape change, and genetic correlation structure in the rodent mandible. Syst. Biol. 41: 196-221.

Atchley, W. R., and Hall, B. K. 1991. A model for development and evolution of complex morphological structures. Biol. Rev. Cambridge Phil. Soc. 66: 101-157.

Atchley, W. R., Plummer, A. A., and Riska, B. 1985. Genetics of mandible form in the mouse. Genetics 111: 555-577.

Auffray, J.-C., Alibert, P., Renaud, S., Orth, A., and Bonhomme, F. 1996. Fluctuating asymmetry in Mus musculus subspecific hybridization: traditional and procrustes comparative approaches. In L. F. Marcus, M. Corti, A. Loy, G. J. P. Naylor, and D. E. Slice (eds.). Advance in Morphometrics. Nato ASI Series, Series A, Life Science. pp. 275-284.

Badyaev, A. V., and Foresman, K. R. 2004. Evolution of morphological integration. I. Functional units channel stress-induced variation in shrew mandibles. Am. Natural. 163: 868-879.

Badyaev, A. V., Foresman, K. R., and Fernandes, M. V. 2000. Stress and developmental stability: vegetation removal causes increased fluctuating asymmetry in shrews. Ecology 81: 336-345.

Badyaev, A. V., Foresman, K. R., and Young, R. L. 2005. Evolution of morphological integration: developmental accommodation of stressinduced variation. Am. Natural. 166: 382-395.

Bolker, J. A. 2000. Modularity in development and why it matters to evo-devo. Am. Zool. 40: 770-776.

Bookstein, F. L. 1997. Landmark methods for forms without landmarks: morphometrics of group differences in outline shape. Med. Image Anal. 1: $97-118$

Bookstein, F. L., Streissguth, A. P., Sampson, P. D., Connor, P. D., and Barr, H. M. 2002. Corpus callosum shape and neuropsychological deficits in adult males with heavy fetal alcohol exposure. Neuroimage 15 : 233-251.

Burr, D. B., Robling, A. G., and Turner, C. H. 2002. Effects of biomechanical stress on bones in animals. Bone 30: 781-786.

Carter, D. R., Van der Meulen, M. C. H., and Beaupre, G. S. 1996. Mechanical factors in bone growth and development. Bone 18: S5-S10.

Cheverud, J. M. 1982. Phenotypic, genetic, and environmental morphological integration in the cranium. Evolution 36: 499-516. 
Cheverud, J. M. 1984. Quantitative genetics and developmental constraints on evolution by selection. J. Theoret. Biol. 110: 155-171.

Cheverud, J. M. 1995. Morphological integration in the saddle-back tamarin (Saguinus fuscicollis) cranium. Am. Natural. 145: 63-89.

Cheverud, J. M. 1996. Developmental integration and the evolution of pleiotropy. Am. Zool. 36: 44-50.

Cheverud, J. M. 2004. Modular pleiotropic effects of quantitative trait loci on morphological traits. Modul. Dev. Evol. 132-153.

Cheverud, J. M., Hartman, S. E., Richtsmeier, J. T., and Atchley, W. R. 1991. A quantitative genetic analysis of localized morphology in mandibles of inbred mice using finite-element scaling analysis. J. Craniofac. Genet. Dev. Biol. 11: 122-137.

Dietz, E. J. 1983. Permutation tests for association between 2 distance matrices. Syst. Zool. 32: 21-26.

Dow, M. M., and Cheverud, J. M. 1985. Comparison of cistance matrices in studies of population-structure and genetic microdifferentiation Quadratic assignment. Am. J. Phys. Anthropol. 68: 367-373.

Farris, J. S. 1969. On the cophenetic correlation coefficient. Syst. Zool. 18: $279-285$.

Gerhart, J., and Kirschner, M. 2007. The theory of facilitated variation. Proc. Natl. Acad. Sci. USA 104: 8582-8589.

Goswami, A. 2006. Cranial modularity shifts during mammalian evolution. Am. Natural. 168: 270-280.

Green, W. D. K. 1996. The thin-plate spline and images with curving features. In K. V. Mardia, C. A. Gill, and I. L. Dryden (eds.). Image Fusion and Shape Variability. University of Leeds Press, Leeds, pp. 79-87.

Gunz, P., Mitteroecker, P., and Bookstein, F. L. 2005. Semilandmarks in three dimensions. In D. E. Slice (ed.). Modern Morphometrics in Physical Anthropology. Kluwer Academic/Plenum Publishers, New York, pp. 73-98.

Hall, B. K. 2003. Unlocking the black box between genotype and phenotype: cell condensations as morphogenetic (modular) units. Biol. Phil. 18 219-247.

Hall, B. K., and Herring, S. W. 1990. Paralysis and growth of the musculoskeletal system in the embryonic chick. J. Morphol. 206: 45-56.

Hall, B. K., and Miyake, T. 2000. All for one and one for all: condensations and the initiation of skeletal development. Bioessays 22: 138-147.

Hallgrímsson, B., Dorval, C. J., Zelditch, M. L., and German, R. Z. 2004. Craniofacial variability and morphological integration in mice susceptible to cleft lip and palate. J. Anat. 205: 501-517.

Hallgrímsson, B., Lieberman, D. E., Liu, W., Ford-Hutchinson, A. F., and Jirik, F. R. 2007a. Epigenetic interactions and the structure of phenotypic variation in the cranium. Evol. Dev. 9: 76-91

Hallgrímsson, B., Lieberman, D. E., Young, N. M., Parsons, T., and Wat, S. 2007b. Evolution of covariance in the mammalian skull. Novartis Found Symp. 284: 164-185; discussion 185-190.

Hallgrímsson, B., Willmore, K., and Hall, B. K. 2002. Canalization, developmental stability, and morphological integration in primate limbs. Yearbook Phys. Anthropol. 45: 131-158.

Harris, S. E., et al. 2004. Mapping expression patterns of mechanically responsive genes, DMP1 and MEPE, in osteocytes using the mouse ulnae: correlation to predicted local strain. J. Bone Mineral Res. 19: S28S28.

Henderson, J. H., Longaker, M. T., and Carter, D. R. 2004. Sutural bone deposition rate and strain magnitude during cranial development. Bone 34: $271-280$

Herring, S. W. 1993. Formation of the vertebrate face: epigenetic and functional influences. Am. Zool. 33: 472-483.

Herring, S. W. 1994. Development of functional interactions between skeletal and muscular systems. In B. K. Hall (ed.). Bone Vol. 9: Differentiation and Morphogenesis of Bone. CRC Press Inc., Boca Raton, pp. 165-191.

Herring, S. W., and Lakars, T. C. 1981. Craniofacial development in the absence of muscle contraction. J. craniofac. Genet. Dev. Biol. 1: 341-357.

Hood, G. M. 2008. PopTools version 3.0.3. URL: http://www.cse.csiro.au/ poptools

Jojic, V., Blagojevic, J., Ivanovic, A., Bugarski-Stanojevic, V., and Vujosevic, M. 2007. Morphological integration of the mandible in yellownecked field mice: the effects of B chromosomes. J. Mammal. 88: 689-695.
Kaufman, L., and Rousseeuw, P. 1990. Finding Groups in Data, an Introduction to Cluster Analysis. Wiley, New York.

Klingenberg, C. P. 2004. Integration, modules, and development Molecules to morphology to evolution. Phenotypic Integration: Studying the Ecology and Evolution of Complex Phenotypes. Oxford University Press, Oxford, pp. 213-230.

Klingenberg, C. P. 2005. Developmental constraints, modules, and evolvability. In B. Hallgrimsson and B. K. Hall (eds.). Variation: A Central Concept in Biology. Elsevier Academic Press, San Diego, pp. 219-247.

Klingenberg, C. P., Badyaev, A. V., Sowry, S. M., and Beckwith, N. J. 2001. Inferring developmental modularity from morphological integration: analysis of individual variation and asymmetry in bumblebee wings. Am. Natural. 157: 11-23.

Klingenberg, C. P., Barluenga, M., and Meyer, A. 2002. Shape analysis of symmetric structures: quantifying variation among individuals and asymmetry. Evolution 56: 1909-1920.

Klingenberg, C. P., Leamy, L. J., and Cheverud, J. M. 2004. Integration and modularity of quantitative trait locus effects on geometric shape in the mouse mandible. Genetics 166: 1909-1921.

Klingenberg, C. P., and McIntyre, G. S. 1998. Geometric morphometrics of developmental instability: analyzing patterns of fluctuating asymmetry with procrustes methods. Evolution 52: 1363-1375.

Klingenberg, C. P., Mebus, K., and Auffray, J. C. 2003. Developmental integration in a complex morphological structure: how distinct are the modules in the mouse mandible? Evol. Dev. 5: 522-531.

Klingenberg, C. P., and Zaklan, S. D. 2000. Morphological integration between developmental compartments in the Drosophila wing. Evolution 54: $1273-1285$.

Lande, R. 1980. The genetic covariance between characters maintained by pleiotropic mutations. Genetics 94: 203-215.

Leamy, L. 1984. Morphometric studies in inbred and hybrid house mice. 5 Directional and fluctuating asymmetry. Am. Natural. 123: 579-593.

Maechler, M., Rousseeuw, P., Struyf, A., and Hubert, M. 2005. Cluster analysis basics and extensions.

Magwene, P. M. (2001). New tools for studying integration and modularity. Evolution 55: 1734-1745.

Makarenkov, U. 2000. T-Rex, ver. 4.0a1. URL: http://www.labunix. uqam.ca/ makarenv/trex.html

Makarenkov, V., and Legendre, P. 2004. From a phylogenetic tree to a reticulated network. J. Comput. Biol. 11: 195-212.

Makarenkov, V., Legendre, P., and Desdevises, Y. 2004. Modelling phylogenetic relationships using reticulated networks. Zool. Scripta 33: 89-96.

Marquez, E. 2007a. Coriandis. URL: http://www-personal.umich.edu/ $\sim$ emarquez/morph/index.html

Marquez, E. 2007b. Sage, ver. 1.03. URL: http://www-personal.umich.edu/ $\sim$ emarquez/morph/index.html

Mezey, J. G., Cheverud, J. M., and Wagner, G. P. 2000. Is the genotypephenotype map modular: a statistical approach using mouse quantitative trait loci data. Genetics 156: 305-311.

Monteiro, L. R., Bonato, V., and dos Reis, S. F. 2005. Evolutionary integration and morphological diversification in complex morphological structures: mandible shape divergence in spiny rats (Rodentia, Echimyidae). Evol. Dev. 7: 429-439.

Myers, P., Lundrigan, B. L., Gillespie, B. W., and Zelditch, M. L. 1996. Phenotypic plasticity in skull and dental morphology in the prairie deer mouse (Peromyscus maniculatus bairdii). J. Morphol. 229: 229-237.

Needham, J. 1933. On the dissociability of the fundamental process in ontogenesis. Biol. Rev. Cambridge Phil. Soc. 8: 180-223.

Palmer, A. R., and Strobeck, C. 1986. Fluctuating asymmetry: measurement, analysis, patterns. Ann. Rev. Ecol. Syst. 17: 391-421.

Pavlicev, M., Kenney-Hunt, J. P., Norgard, E. A., Roseman, C. C., Wolf, J. B., and Cheverud, J. M. 2008. Genetic variation in pleiotropy: differential epistasis as a source of variation in the allometric relationship between long bone lengths and body weight. Evolution 62: 199-213.

R Development Core Team. 2008. R: A language and environment for statistical computing version 2.6.2. R Foundation for Statistical Computing Vienna, Austria. URL: http://www.r-project.org.

Raff, R. A. 1996. The Shape of Life: Genes, Development and the Evolution of Animal Form. University of Chicago Press, Chicago. 
Richtsmeier, J. T., et al. 2006. Phenotypic integration of neurocranium and brain. J. Exp. Zool. Part B-Mol. Dev. Evol. 306B: 360-378.

Rohlf, F. J. 2006. tpsDig2.1. State University of New York, Stony Brook. URL: http:/life.bio.sunysb.edu/morph

Rot-Nikcevic, I., Downing, K. J., Hall, B. K., and Kablar, B. 2007. Development of the mouse mandibles and clavicles in the absence of skeletal myogenesis. Histol. Histopathol. 22: 51-60.

Rot-Nikcevic, I., et al. 2006. Myf5(-/-): $\mathrm{MyoD}(-/-)$ amyogenic fetuses reveal the importance of early contraction and static loading by striated muscle in mouse skeletogenesis. Dev. Genes Evol. 216: 1-9.

Sampson, P. D., Bookstein, F. L., Sheehan, H., and Bolson, E. L. 1996 Eigenshape analysis of left ventricular outlines from contrast ventriculograms. In L. F. Marcus, M. Corti, A. Loy, G. J. P. Naylor, and D. E. Slice (eds.). Advances in Morphometrics. Nato ASI series, Series A, Life Science. New York, pp. 131-152.

Sattath, S., and Tversky, A. 1977. Additive similarity trees. Psychometrika 42: 319-345.

Sheets, H. D. 2003. Semiland. URL: http://www.canisius3.edu/ sheets/ morphosoft.html.

Sheets, H. D., Kim, K., and Mitchell, C. E. 2004. A combined landmark and outline-based approach to ontogenetic shape change in the Ordovician trilobite Triarthrus becki. In A. M. T. Elewa (ed.). Morphometrics: Applications in Biology and Paleontology. Springer, New York, pp. 67-82.

Sneath, P. H. A., and Sokal, R. R. 1973. Numerical Taxonomy. W. H. Freeman, San Francisco.

Wagner, G. P. 1988. The influence of variation and of developmental constraints on the rate of multivariate phenotypic evolution. J. Evol. Biol. 1: $45-66$.
Wagner, G. P. 1996. Homologues, natural kinds and the evolution of modularity. Am. Zool. 36: 36-43.

Wagner, G. P., and Altenberg, L. 1996. Complex adaptations and the evolution of evolvability. Evolution 50: 967-976.

Wagner, G. P., Mezey, J. G., and Calabretta, R. 2005. Natural selection and the origin of modules. In W. Callebaut and D. Rasskin-Gutman (eds.). Modularity: Understanding the Development and Evolution of Natural Complex Systems. MIT Press, Cambridge, MA, pp. 33-50.

Wagner, G. P., Pavlicev, M., and Cheverud, J. M. 2007. The road to modularity. Nat. Rev. Genet. 8: 921-931.

Ward, J. H. 1963. Hierarchical grouping to optimize an objective function. J. Am. Statis. Assoc. 58: 236-244.

Wilkinson, L. 2000. Systat version 10. SPSS Inc., Chicago, IL.

Willmore, K. E., Zelditch, M. L., Young, N., Ah-Seng, A., Lozanoff, S., and Hallgrimsson, B. 2006. Canalization and developmental stability in the brachyrrhine mouse. J. Anat. 208: 361-372.

Wolf, J. B., Frankino, W. A., Agrawal, A. F., Brodie, E. D., and Moore, A. J. 2001. Developmental interactions and the constituents of quantitative variation. Evolution 55: 232-245.

Young, N. M., and Hallgrimsson, B. 2005. Serial homology and the evolution of mammalian limb covariation structure. Evolution 59: 2691-2704.

Young, R. L., and Badyaev, A. V. 2006. Evolutionary persistence of phenotypic integration: influence of developmental and functional relationships on complex trait evolution. Evolution 60: 1291-1299.

Zelditch, M. L., and Carmichael, A. C. 1989. Ontogenetic variation in patterns of developmental and functional integration in skulls of Sigmodon fulviventer. Evolution 43: 814-824. 